\title{
An Extragradient Method for Mixed Equilibrium Problems and Fixed Point Problems
}

\author{
Yonghong Yao, ${ }^{1}$ Yeong-Cheng Liou, ${ }^{2}$ and Yuh-Jenn $\mathrm{Wu}^{3}$ \\ ${ }^{1}$ Department of Mathematics, Tianjin Polytechnic University, Tianjin 300160, China \\ ${ }^{2}$ Department of Information Management, Cheng Shiu University, Kaohsiung 833, Taiwan \\ ${ }^{3}$ Department of Applied Mathematics, Chung Yuan Christian University, Chung Li 320, Taiwan \\ Correspondence should be addressed to Yeong-Cheng Liou, simplex_liou@hotmail.com \\ Received 2 November 2008; Revised 8 April 2009; Accepted 23 May 2009 \\ Recommended by Nan-Jing Huang
}

The purpose of this paper is to investigate the problem of approximating a common element of the set of fixed points of a demicontractive mapping and the set of solutions of a mixed equilibrium problem. First, we propose an extragradient method for solving the mixed equilibrium problems and the fixed point problems. Subsequently, we prove the strong convergence of the proposed algorithm under some mild assumptions.

Copyright (C) 2009 Yonghong Yao et al. This is an open access article distributed under the Creative Commons Attribution License, which permits unrestricted use, distribution, and reproduction in any medium, provided the original work is properly cited.

\section{Introduction}

Let $H$ be a real Hilbert space and let $C$ be a nonempty closed convex subset of $H$. Let $\varphi$ : $C \rightarrow \mathbf{R}$ be a real-valued function and $\Theta: C \times C \rightarrow \mathbf{R}$ be an equilibrium bifunction, that is, $\Theta(u, u)=0$ for each $u \in C$. We consider the following mixed equilibrium problem (MEP) which is to find $x^{*} \in C$ such that

$$
\Theta\left(x^{*}, y\right)+\varphi(y)-\varphi\left(x^{*}\right) \geq 0, \quad \forall y \in C
$$

In particular, if $\varphi \equiv 0$, this problem reduces to the equilibrium problem (EP), which is to find $x^{*} \in C$ such that

$$
\Theta\left(x^{*}, y\right) \geq 0, \quad \forall y \in C
$$

Denote the set of solutions of (MEP) by $\Omega$ and the set of solutions of (EP) by $\Gamma$. The mixed equilibrium problems include fixed point problems, optimization problems, variational 
inequality problems, Nash equilibrium problems, and the equilibrium problems as special cases; see, for example, [1-5]. Some methods have been proposed to solve the equilibrium problems, see, for example, [5-21].

In 2005, Combettes and Hirstoaga [6] introduced an iterative algorithm of finding the best approximation to the initial data when $\Gamma \neq \emptyset$ and proved a strong convergence theorem. Recently by using the viscosity approximation method S. Takahashi and W. Takahashi [8] introduced another iterative algorithm for finding a common element of the set of solutions of (EP) and the set of fixed points of a nonexpansive mapping in a real Hilbert space. Let $S: C \rightarrow H$ be a nonexpansive mapping and $f: C \rightarrow C$ be a contraction. Starting with arbitrary initial $x_{1} \in H$, define the sequences $\left\{x_{n}\right\}$ and $\left\{u_{n}\right\}$ recursively by

$$
\begin{gathered}
\Theta\left(u_{n}, y\right)+\frac{1}{r_{n}}\left\langle y-u_{n}, u_{n}-x_{n}\right\rangle \geq 0, \quad \forall y \in C, \\
x_{n+1}=\alpha_{n} f\left(x_{n}\right)+\left(1-\alpha_{n}\right) S u_{n}, \quad \forall n \geq 0 .
\end{gathered}
$$

S. Takahashi and W. Takahashi proved that the sequences $\left\{x_{n}\right\}$ and $\left\{u_{n}\right\}$ defined by (TT) converge strongly to $z \in \operatorname{Fix}(S) \cap \Gamma$ with the following restrictions on algorithm parameters $\left\{\alpha_{n}\right\}$ and $\left\{r_{n}\right\}$ :

(i) $\lim _{n \rightarrow \infty} \alpha_{n}=0$ and $\sum_{n=0}^{\infty} \alpha_{n}=\infty$;

(ii) $\liminf _{n \rightarrow \infty} r_{n}>0$;

(iii) (A1): $\sum_{n=0}^{\infty}\left|\alpha_{n+1}-\alpha_{n}\right|<\infty$; and (R1): $\sum_{n=0}^{\infty}\left|r_{n+1}-r_{n}\right|<\infty$.

Subsequently, some iterative algorithms for equilibrium problems and fixed point problems have further developed by some authors. In particular, Zeng and Yao [16] introduced a new hybrid iterative algorithm for mixed equilibrium problems and fixed point problems and Mainge and Moudafi [22] introduced an iterative algorithm for equilibrium problems and fixed point problems.

On the other hand, for solving the equilibrium problem (EP), Moudafi [23] presented a new iterative algorithm and proved a weak convergence theorem. Ceng et al. [24] introduced another iterative algorithm for finding an element of $\operatorname{Fix}(S) \cap \Gamma$. Let $S: C \rightarrow C$ be a $k$-strict pseudocontraction for some $0 \leq k<1$ such that $\operatorname{Fix}(S) \cap \Gamma \neq \emptyset$. For given $x_{1} \in H$, let the sequences $\left\{x_{n}\right\}$ and $\left\{u_{n}\right\}$ be generated iteratively by

$$
\begin{gathered}
\Theta\left(u_{n}, y\right)+\frac{1}{r_{n}}\left\langle y-u_{n}, u_{n}-x_{n}\right\rangle \geq 0, \quad \forall y \in C, \\
x_{n+1}=\alpha_{n} u_{n}+\left(1-\alpha_{n}\right) S u_{n}, \quad \forall n \geq 1
\end{gathered}
$$

where the parameters $\left\{\alpha_{n}\right\}$ and $\left\{r_{n}\right\}$ satisfy the following conditions:

(i) $\left\{\alpha_{n}\right\} \subset[\alpha, \beta]$ for some $\alpha, \beta \in(k, 1)$;

(ii) $\left\{r_{n}\right\} \subset(0, \infty)$ and $\liminf _{n \rightarrow \infty} r_{n}>0$.

Then, the sequences $\left\{x_{n}\right\}$ and $\left\{u_{n}\right\}$ generated by (CAY) converge weakly to an element of $\operatorname{Fix}(S) \cap \Gamma$.

At this point, we should point out that all of the above results are interesting and valuable. At the same time, these results also bring us the following conjectures. 
Questions

(1) Could we weaken or remove the control condition (iii) on algorithm parameters in S. Takahashi and W. Takahashi [8]?

(2) Could we construct an iterative algorithm for $k$-strict pseudocontractions such that the strong convergence of the presented algorithm is guaranteed?

(3) Could we give some proof methods which are different from those in $[8,12,16,24]$ ?

It is our purpose in this paper that we introduce a general iterative algorithm for approximating a common element of the set of fixed points of a demicontractive mapping and the set of solutions of a mixed equilibrium problem. Subsequently, we prove the strong convergence of the proposed algorithm under some mild assumptions. Our results give positive answers to the above questions.

\section{Preliminaries}

Let $H$ be a real Hilbert space with inner product $\langle\cdot, \cdot\rangle$ and norm $\|\cdot\|$. Let $C$ be a nonempty closed convex subset of $H$.

Let $T: C \rightarrow C$ be a mapping. We use Fix $(T)$ to denote the set of the fixed points of $T$. Recall what follows.

(i) $T$ is called demicontractive if there exists a constant $0 \leq k<1$ such that

$$
\left\|T x-x^{*}\right\|^{2} \leq\left\|x-x^{*}\right\|^{2}+k\|x-T x\|^{2}
$$

for all $x \in C$ and $x^{*} \in \operatorname{Fix}(T)$, which is equivalent to

$$
\left\langle x-T x, x-x^{*}\right\rangle \geq \frac{1-k}{2}\|x-T x\|^{2}
$$

For such case, we also say that $T$ is a $k$-demicontractive mapping.

(ii) $T$ is called nonexpansive if

$$
\|T x-T y\| \leq\|x-y\|
$$

for all $x, y \in C$.

(iii) $T$ is called quasi-nonexpansive if

$$
\left\|T x-x^{*}\right\| \leq\left\|x-x^{*}\right\|
$$

for all $x \in C$ and $x^{*} \in \operatorname{Fix}(T)$.

(iv) $T$ is called strictly pseudocontractive if there exists a constant $0 \leq k<1$ such that

$$
\|T x-T y\|^{2} \leq\|x-y\|^{2}+k\|(x-T x)-(y-T y)\|^{2}
$$

for all $x, y \in C$. 
It is worth noting that the class of demicontractive mappings includes the class of the nonexpansive mappings, the quasi-nonexpansive mappings and the strictly pseudocontractive mappings as special cases. we have

Let us also recall that $T$ is called demiclosed if for any sequence $\left\{x_{n}\right\} \subset H$ and $x \in H$,

$$
x_{n} \longrightarrow x \text { weakly, } \quad(I-T) x_{n} \longrightarrow 0 \text { strongly } \Longrightarrow x \in \operatorname{Fix}(T)
$$

It is well-known that the nonexpansive mappings, strictly pseudo-contractive mappings are all demiclosed. See, for example, [25-27].

An operator $A: C \rightarrow H$ is said to be $\delta$-strongly monotone if there exists a positive constant $\delta$ such that

$$
\langle A x-A y, x-y\rangle \geq \delta\|x-y\|^{2}
$$

for all $x, y \in C$.

Now we concern the following problem: find $x^{*} \in \operatorname{Fix}(T) \cap \Omega$ such that

$$
\left\langle A x^{*}, x-x^{*}\right\rangle \geq 0, \quad \forall x \in \operatorname{Fix}(T) \cap \Omega .
$$

In this paper, for solving problem (2.8) with an equilibrium bifunction $\Theta: C \times C \rightarrow \mathbf{R}$, we assume that $\Theta$ satisfies the following conditions:

(H1) $\Theta$ is monotone, that is, $\Theta(x, y)+\Theta(y, x) \leq 0$ for all $x, y \in C$;

(H2) for each fixed $y \in C, x \mapsto \Theta(x, y)$ is concave and upper semicontinuous;

(H3) for each $x \in C, y \mapsto \Theta(x, y)$ is convex.

A mapping $\eta: C \times C \rightarrow H$ is called Lipschitz continuous, if there exists a constant $\ell>0$ such that

$$
\|\eta(x, y)\| \leq \lambda\|x-y\|, \quad \forall x, y \in C
$$

A differentiable function $K: C \rightarrow \mathbf{R}$ on a convex set $C$ is called

(i) $\eta$-convex if

$$
K(y)-K(x) \geq\left\langle K^{\prime}(x), \eta(y, x)\right\rangle, \quad \forall x, y \in C,
$$

where $K^{\prime}$ is the Frechet derivative of $K$ at $x$;

(ii) $\eta$-strongly convex if there exists a constant $\sigma>0$ such that

$$
K(y)-K(x)-\left\langle K^{\prime}(x), \eta(y, x)\right\rangle \geq\left(\frac{\sigma}{2}\right)\|x-y\|^{2}, \quad \forall x, y \in C
$$


Let $C$ be a nonempty closed convex subset of a real Hilbert space $H, \varphi: C \rightarrow \mathbf{R}$ be real-valued function and $\Theta: C \times C \rightarrow \mathbf{R}$ be an equilibrium bifunction. Let $r$ be a positive number. For a given point $x \in C$, the auxiliary problem for (MEP) consists of finding $y \in C$ such that

$$
\Theta(y, z)+\varphi(z)-\varphi(y)+\frac{1}{r}\left\langle K^{\prime}(y)-K^{\prime}(x), \eta(z, y)\right\rangle \geq 0, \quad \forall z \in C
$$

Let $S_{r}: C \rightarrow C$ be the mapping such that for each $x \in C, S_{r}(x)$ is the solution set of the auxiliary problem, that is, $\forall x \in C$,

$$
S_{r}(x)=\left\{y \in C: \Theta(y, z)+\varphi(z)-\varphi(y)+\frac{1}{r}\left\langle K^{\prime}(y)-K^{\prime}(x), \eta(z, y)\right\rangle \geq 0, \forall z \in C\right\} .
$$

We need the following important and interesting result for proving our main results.

Lemma 2.1 ([16, 28]). Let $C$ be a nonempty closed convex subset of a real Hilbert space $H$ and let $\varphi: C \rightarrow \mathbf{R}$ be a lower semicontinuous and convex functional. Let $\Theta: C \times C \rightarrow \mathbf{R}$ be an equilibrium bifunction satisfying conditions (H1)-(H3). Assume what follows.

(i) $\eta: C \times C \rightarrow H$ is Lipschitz continuous with constant $\lambda>0$ such that

(a) $\eta(x, y)+\eta(y, x)=0, \forall x, y \in C$,

(b) $\eta(\cdot, \cdot)$ is affine in the first variable,

(c) for each fixed $y \in C, x \mapsto \eta(y, x)$ is sequentially continuous from the weak topology to the weak topology.

(ii) $K: C \rightarrow \mathbf{R}$ is $\eta$-strongly convex with constant $\sigma>0$ and its derivative $K^{\prime}$ is sequentially continuous from the weak topology to the strong topology.

(iii) For each $x \in C$, there exist a bounded subset $D_{x} \subset C$ and $z_{x} \in C$ such that for any $y \in C \backslash D_{x}$

$$
\Theta\left(y, z_{x}\right)+\varphi\left(z_{x}\right)-\varphi(y)+\frac{1}{r}\left\langle K^{\prime}(y)-K^{\prime}(x), \eta\left(z_{x}, y\right)\right\rangle<0 .
$$

Then there hold the following:

(i) $S_{r}$ is single-valued;

(ii) $S_{r}$ is nonexpansive if $K^{\prime}$ is Lipschitz continuous with constant $v>0$ such that $\sigma \geq \lambda v$ and

$$
\left\langle K^{\prime}\left(x_{1}\right)-K^{\prime}\left(x_{2}\right), \eta\left(u_{1}, u_{2}\right)\right\rangle \geq\left\langle K^{\prime}\left(u_{1}\right)-K^{\prime}\left(u_{2}\right), \eta\left(u_{1}, u_{2}\right)\right\rangle, \quad \forall\left(x_{1}, x_{2}\right) \in C \times C,
$$

where $u_{i}=S_{r}\left(x_{i}\right)$ for $i=1,2$;

(iii) $\operatorname{Fix}\left(S_{r}\right)=\Omega$;

(iv) $\Omega$ is closed and convex. 


\section{Main Results}

Let $H$ be a real Hilbert space, $\varphi: H \rightarrow \mathbf{R}$ be a lower semicontinuous and convex real-valued function, $\Theta: H \times H \rightarrow \mathbf{R}$ be an equilibrium bifunction. Let $A: H \rightarrow H$ be a mapping and $T: H \rightarrow H$ be a mapping. In this section, we first introduce the following new iterative algorithm.

Algorithm 3.1. Let $r$ be a positive parameter. Let $\left\{\lambda_{n}\right\}$ be a sequence in $[0, \infty)$ and $\left\{\alpha_{n}\right\}$ be a sequence in $[0,1)$. Define the sequences $\left\{x_{n}\right\},\left\{y_{n}\right\}$, and $\left\{z_{n}\right\}$ by the following manner:

$$
\begin{gathered}
x_{0} \in C \text { chosen arbitrarily, } \\
\Theta\left(z_{n}, x\right)+\varphi(x)-\varphi\left(z_{n}\right)+\frac{1}{r}\left\langle K^{\prime}\left(z_{n}\right)-K^{\prime}\left(x_{n}\right), \eta\left(x, z_{n}\right)\right\rangle \geq 0, \quad \forall x \in C, \\
y_{n}=z_{n}-\lambda_{n} A z_{n}, \\
x_{n+1}=\left(1-\alpha_{n}\right) y_{n}+\alpha_{n} T y_{n} .
\end{gathered}
$$

Now we give a strong convergence result concerning Algorithm 3.1 as follows.

Theorem 3.2. Let $H$ be a real Hilbert space. Let $\varphi: H \rightarrow \mathbf{R}$ be a lower semicontinuous and convex functional. Let $\Theta: H \times H \rightarrow \mathbf{R}$ be an equilibrium bifunction satisfying conditions (H1)-(H3). Let $A: H \rightarrow H$ be an L-Lipschitz continuous and $\delta$-strongly monotone mapping and $T: H \rightarrow H$ be a demiclosed and $k$-demicontractive mapping such that $\operatorname{Fix}(T) \cap \Omega \neq \emptyset$. Assume what follows.

(i) $\eta: H \times H \rightarrow H$ is Lipschitz continuous with constant $\lambda>0$ such that

(a) $\eta(x, y)+\eta(y, x)=0, \forall x, y \in H$,

(b) $\eta(\cdot, \cdot)$ is affine in the first variable,

(c) for each fixed $y \in H, x \mapsto \eta(y, x)$ is sequentially continuous from the weak topology to the weak topology.

(ii) $K: H \rightarrow \mathbf{R}$ is $\eta$-strongly convex with constant $\sigma>0$ and its derivative $K^{\prime}$ is not only sequentially continuous from the weak topology to the strong topology but also Lipschitz continuous with constant $v>0$ such that $\sigma \geq \lambda$ v.

(iii) For each $x \in H$; there exist a bounded subset $D_{x} \subset H$ and $z_{x} \in H$ such that, for any $y \notin D_{x}$

$$
\Theta\left(y, z_{x}\right)+\varphi\left(z_{x}\right)-\varphi(y)+\frac{1}{r}\left\langle K^{\prime}(y)-K^{\prime}(x), \eta\left(z_{x}, y\right)\right\rangle<0 .
$$

(iv) $\alpha_{n} \in[\gamma,(1-k) / 2]$ for some $\gamma>0, \lim _{n \rightarrow \infty} \lambda_{n}=0$ and $\sum_{n=0}^{\infty} \lambda_{n}=\infty$.

Then the sequences $\left\{x_{n}\right\},\left\{y_{n}\right\}$, and $\left\{z_{n}\right\}$ generated by (3.1) converge strongly to $x^{*}$ which solves the problem (2.8) provided $S_{r}$ is firmly nonexpansive. 
Proof. First, we prove that $\left\{x_{n}\right\},\left\{y_{n}\right\}$, and $\left\{z_{n}\right\}$ are all bounded. Without loss of generality, we may assume that $0<\delta<L$. Given $\mu \in\left(0,2 \delta / L^{2}\right)$ and $x, y \in H$, we have

$$
\begin{aligned}
\|(\mu A-I) x-(\mu A-I) y\|^{2} & =\mu^{2}\|A x-A y\|^{2}+\|x-y\|^{2}-2 \mu\langle A x-A y, x-y\rangle \\
& \leq \mu^{2} L^{2}\|x-y\|^{2}+\|x-y\|^{2}-2 \mu \delta\|x-y\|^{2} \\
& =\left(1-2 \mu \delta+\mu^{2} L^{2}\right)\|x-y\|^{2},
\end{aligned}
$$

that is,

$$
\|(\mu A-I) x-(\mu A-I) y\| \leq \sqrt{1-2 \mu \delta+\mu^{2} L^{2}}\|x-y\| .
$$

Take $x^{*} \in \operatorname{Fix}(T) \cap \Omega$. From (3.1), we have

$$
\begin{aligned}
\left\|y_{n+1}-\left(x^{*}-\lambda_{n+1} A x^{*}\right)\right\| & =\left\|\left(z_{n+1}-\lambda_{n+1} A z_{n+1}\right)-\left(x^{*}-\lambda_{n+1} A x^{*}\right)\right\| \\
& =\left\|\left(1-\frac{\lambda_{n+1}}{\mu}\right)\left(z_{n+1}-x^{*}\right)-\frac{\lambda_{n+1}}{\mu}\left((\mu A-I) z_{n+1}-(\mu A-I) x^{*}\right)\right\| \\
& \leq\left(1-\frac{\lambda_{n+1}}{\mu}\right)\left\|z_{n+1}-x^{*}\right\|+\frac{\lambda_{n+1}}{\mu}\left\|(\mu A-I) z_{n+1}-(\mu A-I) x^{*}\right\| .
\end{aligned}
$$

Therefore,

$$
\left\|y_{n+1}-\left(x^{*}-\lambda_{n+1} A x^{*}\right)\right\| \leq\left(1-\frac{\lambda_{n+1} \omega}{\mu}\right)\left\|z_{n+1}-x^{*}\right\|
$$

where $\omega=1-\sqrt{1-2 \mu \delta+\mu^{2} L^{2}} \in(0,1)$.

Note that $z_{n+1}=S_{r} x_{n+1}$ and $S_{r}$ are firmly nonexpansive. Hence, we have

$$
\begin{aligned}
\left\|z_{n+1}-x^{*}\right\|^{2} & =\left\|S_{r} x_{n+1}-S_{r} x^{*}\right\|^{2} \\
& \leq\left\langle S_{r} x_{n+1}-S_{r} x^{*}, x_{n+1}-x^{*}\right\rangle \\
& =\left\langle z_{n+1}-x^{*}, x_{n+1}-x^{*}\right\rangle \\
& =\frac{1}{2}\left(\left\|z_{n+1}-x^{*}\right\|^{2}+\left\|x_{n+1}-x^{*}\right\|^{2}-\left\|x_{n+1}-z_{n+1}\right\|^{2}\right),
\end{aligned}
$$

which implies that

$$
\left\|z_{n+1}-x^{*}\right\|^{2} \leq\left\|x_{n+1}-x^{*}\right\|^{2}-\left\|x_{n+1}-z_{n+1}\right\|^{2}
$$


From (2.2) and (3.1), we have

$$
\begin{aligned}
\left\|x_{n+1}-x^{*}\right\|^{2} & =\left\|\left(1-\alpha_{n}\right) y_{n}+\alpha_{n} T y_{n}-x^{*}\right\|^{2} \\
& =\left\|\left(y_{n}-x^{*}\right)-\alpha_{n}\left(y_{n}-T y_{n}\right)\right\|^{2} \\
& =\left\|y_{n}-x^{*}\right\|^{2}-2 \alpha_{n}\left\langle y_{n}-T y_{n}, y_{n}-x^{*}\right\rangle+\alpha_{n}^{2}\left\|y_{n}-T y_{n}\right\|^{2} \\
& \leq\left\|y_{n}-x^{*}\right\|^{2}-2 \alpha_{n} \frac{1-k}{2}\left\|y_{n}-T y_{n}\right\|^{2}+\alpha_{n}^{2}\left\|y_{n}-T y_{n}\right\|^{2} \\
& =\left\|y_{n}-x^{*}\right\|^{2}-\alpha_{n}\left(1-k-\alpha_{n}\right)\left\|y_{n}-T y_{n}\right\|^{2} \\
& \leq\left\|y_{n}-x^{*}\right\|^{2} .
\end{aligned}
$$

From (3.6)-(3.9), we have

$$
\begin{aligned}
\left\|y_{n+1}-x^{*}\right\| & \leq\left\|y_{n+1}-\left(x^{*}-\lambda_{n+1} A x^{*}\right)\right\|+\lambda_{n+1}\left\|A x^{*}\right\| \\
& \leq\left(1-\frac{\lambda_{n+1} \omega}{\mu}\right)\left\|z_{n+1}-x^{*}\right\|+\lambda_{n+1}\left\|A x^{*}\right\| \\
& \leq\left(1-\frac{\lambda_{n+1} \omega}{\mu}\right)\left\|x_{n+1}-x^{*}\right\|+\lambda_{n+1}\left\|A x^{*}\right\| \\
& \leq\left(1-\frac{\lambda_{n+1} \omega}{\mu}\right)\left\|y_{n}-x^{*}\right\|+\lambda_{n+1}\left\|A x^{*}\right\| \\
& =\left(1-\frac{\lambda_{n+1} \omega}{\mu}\right)\left\|y_{n}-x^{*}\right\|+\frac{\lambda_{n+1} \omega}{\mu}\left\{\frac{\mu}{\omega}\left\|A x^{*}\right\|\right\} \\
& \leq \max \left\{\left\|y_{n}-x^{*}\right\|, \frac{\mu\left\|A x^{*}\right\|}{\omega}\right\} \\
& \leq \cdots \\
& \leq \max \left\{\left\|y_{0}-x^{*}\right\|, \frac{\mu\left\|A x^{*}\right\|}{\omega}\right\} .
\end{aligned}
$$

This implies that $\left\{y_{n}\right\}$ is bounded, so are $\left\{x_{n}\right\}$ and $\left\{z_{n}\right\}$.

From (3.1), we can write $y_{n}-T y_{n}=\left(1 / \alpha_{n}\right)\left(y_{n}-x_{n+1}\right)$. Thus, from (3.9), we have

$$
\begin{aligned}
\left\|x_{n+1}-x^{*}\right\|^{2} & \leq\left\|y_{n}-x^{*}\right\|^{2}-\alpha_{n}\left(1-k-\alpha_{n}\right)\left\|y_{n}-T y_{n}\right\|^{2} \\
& \leq\left\|y_{n}-x^{*}\right\|^{2}-\frac{1-k-\alpha_{n}}{\alpha_{n}}\left\|y_{n}-x_{n+1}\right\|^{2} .
\end{aligned}
$$


Since $\alpha_{n} \in(0,(1-k) / 2],\left(1-k-\alpha_{n}\right) / \alpha_{n} \geq 1$. Therefore, from (3.8) and (3.11), we obtain

$$
\begin{aligned}
\left\|x_{n+1}-x^{*}\right\|^{2} \leq & \left\|y_{n}-x^{*}\right\|^{2}-\left\|y_{n}-x_{n+1}\right\|^{2} \\
= & \left\|z_{n}-x^{*}-\lambda_{n} A z_{n}\right\|^{2}-\left\|z_{n}-x_{n+1}-\lambda_{n} A z_{n}\right\|^{2} \\
= & \left\|z_{n}-x^{*}\right\|^{2}-2 \lambda_{n}\left\langle A z_{n}, z_{n}-x^{*}\right\rangle+\lambda_{n}^{2}\left\|A z_{n}\right\|^{2} \\
& \quad-\left\|z_{n}-x_{n+1}\right\|^{2}+2 \lambda_{n}\left\langle A z_{n}, z_{n}-x_{n+1}\right\rangle-\lambda_{n}^{2}\left\|A z_{n}\right\|^{2} \\
= & \left\|z_{n}-x^{*}\right\|^{2}-2 \lambda_{n}\left\langle x_{n+1}-x^{*}, A z_{n}\right\rangle-\left\|x_{n+1}-z_{n}\right\|^{2} \\
\leq & \left\|x_{n}-x^{*}\right\|^{2}-\left\|x_{n}-z_{n}\right\|^{2}-2 \lambda_{n}\left\langle x_{n+1}-x^{*}, A z_{n}\right\rangle-\left\|x_{n+1}-z_{n}\right\|^{2} .
\end{aligned}
$$

We note that $\left\{x_{n}\right\}$ and $\left\{z_{n}\right\}$ are bounded. So there exists a constant $M \geq 0$ such that

$$
\left|\left\langle x_{n+1}-x^{*}, A z_{n}\right\rangle\right| \leq M \quad \forall n \geq 0 .
$$

Consequently, we get

$$
\left\|x_{n+1}-x^{*}\right\|^{2}-\left\|x_{n}-x^{*}\right\|^{2}+\left\|x_{n+1}-z_{n}\right\|^{2}+\left\|x_{n}-z_{n}\right\|^{2} \leq 2 M \lambda_{n} .
$$

Now we divide two cases to prove that $\left\{x_{n}\right\}$ converges strongly to $x^{*}$.

Case 1. Assume that the sequence $\left\{\left\|x_{n}-x^{*}\right\|\right\}$ is a monotone sequence. Then $\left\{\left\|x_{n}-x^{*}\right\|\right\}$ is convergent. Setting $\lim _{n \rightarrow \infty}\left\|x_{n}-x^{*}\right\|=d$.

(i) If $d=0$, then the desired conclusion is obtained.

(ii) Assume that $d>0$. Clearly, we have

$$
\left\|x_{n+1}-x^{*}\right\|^{2}-\left\|x_{n}-x^{*}\right\|^{2} \longrightarrow 0,
$$

this together with $\lambda_{n} \rightarrow 0$ and (3.14) implies that

$$
\left\|x_{n+1}-z_{n}\right\|^{2}+\left\|x_{n}-z_{n}\right\|^{2} \longrightarrow 0
$$

that is to say

$$
\left\|x_{n+1}-z_{n}\right\| \longrightarrow 0, \quad\left\|x_{n}-z_{n}\right\| \longrightarrow 0
$$

Let $z \in H$ be a weak limit point of $\left\{z_{n_{k}}\right\}$. Then there exists a subsequence of $\left\{z_{n_{k}}\right\}$, still denoted by $\left\{z_{n_{k}}\right\}$ which weakly converges to $z$. Noting that $\lambda_{n} \rightarrow 0$, we also have

$$
y_{n_{k}}=z_{n_{k}}-\lambda_{n_{k}} A z_{n_{k}} \longrightarrow z \text { weakly. }
$$


Combining (3.1) and (3.17), we have

$$
\begin{aligned}
\left\|T y_{n_{k}}-y_{n_{k}}\right\| & =\frac{1}{\alpha_{n_{k}}}\left\|y_{n_{k}}-x_{n_{k}+1}\right\| \\
& =\frac{1}{\alpha_{n_{k}}}\left\|x_{n_{k}+1}-z_{n_{k}}+\lambda_{n_{k}} A z_{n_{k}}\right\| \\
& \leq\left\|x_{n_{k}+1}-z_{n_{k}}\right\|+\lambda_{n_{k}}\left\|A z_{n_{k}}\right\| \\
& \longrightarrow 0
\end{aligned}
$$

Since $T$ is demiclosed, then we obtain $z \in \operatorname{Fix}(T)$.

Next we show that $z \in \Omega$. Since $z_{n}=S_{r} x_{n}$, we derive

$$
\Theta\left(z_{n}, x\right)+\varphi(x)-\varphi\left(z_{n}\right)+\frac{1}{r}\left\langle K^{\prime}\left(z_{n}\right)-K^{\prime}\left(x_{n}\right), \eta\left(x, z_{n}\right)\right\rangle \geq 0, \quad \forall x \in C .
$$

From the monotonicity of $\Theta$, we have

$$
\frac{1}{r}\left\langle K^{\prime}\left(z_{n}\right)-K^{\prime}\left(x_{n}\right), \eta\left(x, z_{n}\right)\right\rangle+\varphi(x)-\varphi\left(z_{n}\right) \geq-\Theta\left(z_{n}, x\right) \geq \Theta\left(x, z_{n}\right),
$$

and hence

$$
\left\langle\frac{K^{\prime}\left(z_{n_{k}}\right)-K^{\prime}\left(x_{n_{k}}\right)}{r}, \eta\left(x, z_{n_{k}}\right)\right\rangle+\varphi(x)-\varphi\left(z_{n_{k}}\right) \geq \Theta\left(x, z_{n_{k}}\right) .
$$

Since $\left(K^{\prime}\left(z_{n_{k}}\right)-K^{\prime}\left(x_{n_{k}}\right)\right) / r \rightarrow 0$ and $z_{n_{k}} \rightarrow z$ weakly, from the weak lower semicontinuity of $\varphi$ and $\Theta(x, y)$ in the second variable $y$, we have

$$
\Theta(x, z)+\varphi(z)-\varphi(x) \leq 0
$$

for all $x \in C$. For $0<t \leq 1$ and $x \in C$, let $x_{t}=t x+(1-t) z$. Since $x \in C$ and $z \in C$, we have $x_{t} \in C$ and hence $\Theta\left(x_{t}, z\right)+\varphi(z)-\varphi\left(x_{t}\right) \leq 0$. From the convexity of equilibrium bifunction $\Theta(x, y)$ in the second variable $y$, we have

$$
\begin{aligned}
0 & =\Theta\left(x_{t}, x_{t}\right)+\varphi\left(x_{t}\right)-\varphi\left(x_{t}\right) \\
& \leq t \Theta\left(x_{t}, x\right)+(1-t) \Theta\left(x_{t}, z\right)+t \varphi(x)+(1-t) \varphi(z)-\varphi\left(x_{t}\right) \\
& \leq t\left[\Theta\left(x_{t}, x\right)+\varphi(x)-\varphi\left(x_{t}\right)\right]
\end{aligned}
$$

and hence $\Theta\left(x_{t}, x\right)+\varphi(x)-\varphi\left(x_{t}\right) \geq 0$. Then, we have

$$
\Theta(z, x)+\varphi(x)-\varphi(z) \geq 0
$$

for all $x \in C$ and hence $z \in \Omega$. 
Fixed Point Theory and Applications

Therefore, we have

$$
z \in \operatorname{Fix}(T) \cap \Omega \text {. }
$$

Thus, if $x^{*}$ is a solution of problem (2.8), we have

$$
\liminf _{k \rightarrow \infty}\left\langle z_{n_{k}}-x^{*}, A x^{*}\right\rangle=\left\langle z-x^{*}, A x^{*}\right\rangle \geq 0 .
$$

Suppose that there exists another subsequence $\left\{z_{n_{i}}\right\}$ which weakly converges to $z^{\prime}$. It is easily checked that $z^{\prime} \in \operatorname{Fix}(T) \cap \Omega$ and

$$
\liminf _{i \rightarrow \infty}\left\langle z_{n_{i}}-x^{*}, A x^{*}\right\rangle=\left\langle z^{\prime}-x^{*}, A x^{*}\right\rangle \geq 0 .
$$

Therefor, we have

$$
\liminf _{n \rightarrow \infty}\left\langle z_{n}-x^{*}, A x^{*}\right\rangle \geq 0 .
$$

Since $A$ is $\delta$-strongly monotone, we have

$$
\left\langle x_{n+1}-x^{*}, A z_{n}\right\rangle \geq \delta\left\|z_{n}-x^{*}\right\|^{2}+\left\langle z_{n}-x^{*}, A x^{*}\right\rangle+\left\langle x_{n+1}-z_{n}, A z_{n}\right\rangle \text {. }
$$

By (3.17)-(3.30), we get

$$
\liminf _{n \rightarrow \infty}\left\langle x_{n+1}-x^{*}, A z_{n}\right\rangle \geq \delta d^{2}
$$

From (3.12), for $0<\epsilon<\delta d^{2}$, we deduce that there exists a positive integer number $n_{0}$ large enough, when $n \geq n_{0}$,

$$
\left\|x_{n+1}-x^{*}\right\|^{2}-\left\|x_{n}-x^{*}\right\|^{2} \leq-2 \lambda_{n}\left(\delta d^{2}-\epsilon\right) .
$$

This implies that

$$
\left\|x_{n+1}-x^{*}\right\|^{2}-\left\|x_{n_{0}}-x^{*}\right\|^{2} \leq-2\left(\delta d^{2}-\epsilon\right) \sum_{k=n_{0}}^{n} \lambda_{k} .
$$

Since $\sum_{n=0}^{\infty} \lambda_{n}=\infty$ and $\left\{x_{n}\right\}$ is bounded, hence the last inequality is a contraction. Therefore, $d=0$, that is to say, $x_{n} \rightarrow x^{*}$.

Case 2. Assume that $\left\{\left\|x_{n}-x^{*}\right\|\right\}$ is not a monotone sequence. Set $\Gamma_{n}=\left\|x_{n}-x^{*}\right\|^{2}$ and let $\tau: N \rightarrow N$ be a mapping for all $n \geq n_{0}$ by

$$
\tau(n)=\max \left\{k \in N: k \leq n, \Gamma_{k} \leq \Gamma_{k+1}\right\} .
$$


Clearly, $\tau$ is a nondecreasing sequence such that $\tau(n) \rightarrow \infty$ as $n \rightarrow \infty$ and $\Gamma_{\tau(n)} \leq \Gamma_{\tau(n)+1}$ for $n \geq n_{0}$. From (3.14), we have

$$
\left\|x_{\tau(n)+1}-z_{\tau(n)}\right\|^{2}+\left\|x_{\tau(n)}-z_{\tau(n)}\right\|^{2} \leq 2 M \lambda_{\tau(n)} \longrightarrow 0,
$$

thus

$$
\left\|x_{\tau(n)+1}-z_{\tau(n)}\right\| \longrightarrow 0, \quad\left\|x_{\tau(n)}-z_{\tau(n)}\right\| \longrightarrow 0
$$

Therefore,

$$
\left\|x_{\tau(n)+1}-x_{\tau(n)}\right\| \longrightarrow 0
$$

Since $\Gamma_{\tau(n)} \leq \Gamma_{\tau(n)+1}$, for all $n \geq n_{0}$, from (3.12), we get

$$
\begin{aligned}
0 & \leq\left\|x_{\tau(n)+1}-x^{*}\right\|^{2}-\left\|x_{\tau(n)}-x^{*}\right\|^{2}+\left\|x_{\tau(n)+1}-z_{\tau(n)}\right\|^{2}+\left\|x_{\tau(n)}-z_{\tau(n)}\right\|^{2} \\
& \leq-2 \lambda_{\tau(n)}\left\langle x_{\tau(n)+1}-x^{*}, A z_{\tau(n)}\right\rangle
\end{aligned}
$$

which implies that

$$
\left\langle x_{\tau(n)+1}-x^{*}, A z_{\tau(n)}\right\rangle \leq 0 \quad \forall n \geq n_{0} .
$$

Since $\left\{z_{\tau(n)}\right\}$ is bounded, there exists a subsequence of $\left\{z_{\tau(n)}\right\}$, still denoted by $\left\{z_{\tau(n)}\right\}$ which converges weakly to $q \in H$. It is easily checked that $q \in \operatorname{Fix}(T) \cap \Omega$. Furthermore, we observe that

$$
\begin{aligned}
\delta\left\|z_{\tau(n)}-x^{*}\right\|^{2} & \leq\left\langle z_{\tau(n)}-x^{*}, A z_{\tau(n)}-A x^{*}\right\rangle \\
& =\left\langle x_{\tau(n)+1}-x^{*}, A z_{\tau(n)}\right\rangle+\left\langle z_{\tau(n)}-x_{\tau(n)+1}, A z_{\tau(n)}\right\rangle-\left\langle z_{\tau(n)}-x^{*}, A x^{*}\right\rangle .
\end{aligned}
$$

Hence, for all $n \geq n_{0}$,

$$
\delta\left\|z_{\tau(n)}-x^{*}\right\|^{2} \leq\left\langle z_{\tau(n)}-x_{\tau(n)+1}, A z_{\tau(n)}\right\rangle-\left\langle z_{\tau(n)}-x^{*}, A x^{*}\right\rangle .
$$

Therefore

$$
\limsup _{n \rightarrow \infty}\left\|z_{\tau(n)}-x^{*}\right\|^{2} \leq-\frac{1}{\delta}\left\langle q-x^{*}, A x^{*}\right\rangle \leq 0,
$$

which implies that

$$
\lim _{n \rightarrow \infty}\left\|z_{\tau(n)}-x^{*}\right\|=0
$$


Thus,

$$
\lim _{n \rightarrow \infty}\left\|x_{\tau(n)}-x^{*}\right\|=0
$$

It is immediate that

$$
\lim _{n \rightarrow \infty} \Gamma_{\tau(n)}=\lim _{n \rightarrow \infty} \Gamma_{\tau(n)+1}=0 .
$$

Furthermore, for $n \geq n_{0}$, it is easily observed that $\Gamma_{n} \leq \Gamma_{\tau(n)+1}$ if $n \neq \tau(n)$ (i.e., $\left.\tau(n)<n\right)$, because $\Gamma_{j}>\Gamma_{j+1}$ for $\tau(n)+1 \leq j \leq n$. As a consequence, we obtain for all $n \geq n_{0}$,

$$
0 \leq \Gamma_{n} \leq \max \left\{\Gamma_{\tau(n)}, \Gamma_{\tau(n)+1}\right\}=\Gamma_{\tau(n)+1}
$$

Hence $\lim _{n \rightarrow \infty} \Gamma_{n}=0$, that is, $\left\{x_{n}\right\}$ converges strongly to $x^{*}$. Consequently, it easy to prove that $\left\{y_{n}\right\}$ and $\left\{z_{n}\right\}$ converge strongly to $x^{*}$. This completes the proof.

Remark 3.3. The advantages of these results in this paper are that less restrictions on the parameters $\left\{\lambda_{n}\right\}$ are imposed.

As direct consequence of Theorem 3.2, we obtain the following.

Corollary 3.4. Let $H$ be a real Hilbert space. Let $\varphi: H \rightarrow \mathbf{R}$ be a lower semicontinuous and convex functional. Let $\Theta: H \times H \rightarrow \mathbf{R}$ be an equilibrium bifunction satisfying conditions (H1)-(H3). Let $A: H \rightarrow H$ be an L-Lipschitz continuous and $\delta$-strongly monotone mapping and $T: H \rightarrow H$ be a nonexpansive mapping such that $\operatorname{Fix}(T) \cap \Omega \neq \emptyset$. Assume what follows.

(i) $\eta: H \times H \rightarrow H$ is Lipschitz continuous with constant $\lambda>0$ such that;

(a) $\eta(x, y)+\eta(y, x)=0, \forall x, y \in H$,

(b) $\eta(\cdot, \cdot)$ is affine in the first variable,

(c) for each fixed $y \in H, x \mapsto \eta(y, x)$ is sequentially continuous from the weak topology to the weak topology.

(ii) $K: H \rightarrow \mathbf{R}$ is $\eta$-strongly convex with constant $\sigma>0$ and its derivative $K^{\prime}$ is not only sequentially continuous from the weak topology to the strong topology but also Lipschitz continuous with constant $v>0$ such that $\sigma \geq \lambda \mathcal{~ v}$.

(iii) For each $x \in H$; there exist a bounded subset $D_{x} \subset H$ and $z_{x} \in H$ such that, for any $y \notin D_{x}$

$$
\Theta\left(y, z_{x}\right)+\varphi\left(z_{x}\right)-\varphi(y)+\frac{1}{r}\left\langle K^{\prime}(y)-K^{\prime}(x), \eta\left(z_{x}, y\right)\right\rangle<0 .
$$

(iv) $\alpha_{n} \in[\gamma,(1-k) / 2]$ for some $\gamma>0, \lim _{n \rightarrow \infty} \lambda_{n}=0$ and $\sum_{n=0}^{\infty} \lambda_{n}=\infty$.

Then the sequences $\left\{x_{n}\right\},\left\{y_{n}\right\}$, and $\left\{z_{n}\right\}$ generated by (3.1) converge strongly to $x^{*}$ which solves the problem (2.8) provided $S_{r}$ is firmly nonexpansive. 
Corollary 3.5. Let $H$ be a real Hilbert space. Let $\varphi: H \rightarrow \mathbf{R}$ be a lower semicontinuous and convex functional. Let $\Theta: H \times H \rightarrow \mathbf{R}$ be an equilibrium bifunction satisfying conditions (H1)-(H3). Let $A: H \rightarrow H$ be an L-Lipschitz continuous and $\delta$-strongly monotone mapping and $T: H \rightarrow H$ be a strictly pseudo-contractive mapping such that $\operatorname{Fix}(T) \cap \Omega \neq \emptyset$. Assume what follows.

(i) $\eta: H \times H \rightarrow H$ is Lipschitz continuous with constant $\lambda>0$ such that

(a) $\eta(x, y)+\eta(y, x)=0, \forall x, y \in H$,

(b) $\eta(\cdot, \cdot)$ is affine in the first variable,

(c) for each fixed $y \in H, x \mapsto \eta(y, x)$ is sequentially continuous from the weak topology to the weak topology.

(ii) $K: H \rightarrow \mathbf{R}$ is $\eta$-strongly convex with constant $\sigma>0$ and its derivative $K^{\prime}$ is not only sequentially continuous from the weak topology to the strong topology but also Lipschitz continuous with constant $v>0$ such that $\sigma \geq \lambda$ v.

(iii) For each $x \in H$; there exist a bounded subset $D_{x} \subset H$ and $z_{x} \in H$ such that, for any $y \notin D_{x}$

$$
\Theta\left(y, z_{x}\right)+\varphi\left(z_{x}\right)-\varphi(y)+\frac{1}{r}\left\langle K^{\prime}(y)-K^{\prime}(x), \eta\left(z_{x}, y\right)\right\rangle<0 .
$$

(iv) $\alpha_{n} \in[\gamma,(1-k) / 2]$ for some $\gamma>0, \lim _{n \rightarrow \infty} \lambda_{n}=0$ and $\sum_{n=0}^{\infty} \lambda_{n}=\infty$.

Then the sequences $\left\{x_{n}\right\},\left\{y_{n}\right\}$ and $\left\{z_{n}\right\}$ generated by (3.1) converge strongly to $x^{*}$ which solves the problem (2.8) provided $S_{r}$ is firmly nonexpansive.

\section{Acknowledgments}

The authors are extremely grateful to the anonymous referee for his/her useful comments and suggestions. The first author was partially supposed by National Natural Science Foundation of China Grant 10771050. The second author was partially supposed by the Grant NSC 97-2221-E-230-017.

\section{References}

[1] E. Blum and W. Oettli, "From optimization and variational inequalities to equilibrium problems," The Mathematics Student, vol. 63, pp. 123-145, 1994.

[2] L.-C. Zeng, S.-Y. Wu, and J.-C. Yao, "Generalized KKM theorem with applications to generalized minimax inequalities and generalized equilibrium problems," Taiwanese Journal of Mathematics, vol. 10, no. 6, pp. 1497-1514, 2006.

[3] O. Chadli, N. C. Wong, and J.-C. Yao, "Equilibrium problems with applications to eigenvalue problems," Journal of Optimization Theory and Applications, vol. 117, no. 2, pp. 245-266, 2003.

[4] O. Chadli, S. Schaible, and J.-C. Yao, "Regularized equilibrium problems with application to noncoercive hemivariational inequalities," Journal of Optimization Theory and Applications, vol. 121, no. 3, pp. 571-596, 2004.

[5] I. V. Konnov, S. Schaible, and J.-C. Yao, "Combined relaxation method for mixed equilibrium problems," Journal of Optimization Theory and Applications, vol. 126, no. 2, pp. 309-322, 2005.

[6] P. L. Combettes and S. A. Hirstoaga, "Equilibrium programming in Hilbert spaces," Journal of Nonlinear and Convex Analysis, vol. 6, no. 1, pp. 117-136, 2005. 
[7] S. D. Flåm and A. S. Antipin, "Equilibrium programming using proximal-like algorithms," Mathematical Programming, vol. 78, no. 1, pp. 29-41, 1997.

[8] S. Takahashi and W. Takahashi, "Viscosity approximation methods for equilibrium problems and fixed point problems in Hilbert spaces," Journal of Mathematical Analysis and Applications, vol. 331, no. 1, pp. 506-515, 2007.

[9] O. Chadli, I. V. Konnov, and J.-C. Yao, "Descent methods for equilibrium problems in a banach space," Computers and Mathematics with Applications, vol. 48, no. 3-4, pp. 609-616, 2004.

[10] X.-P. Ding, Y.-C. Lin, and J.-C. Yao, "Predictor-corrector algorithms for solving generalized mixed implicit quasi-equilibrium problems," Applied Mathematics and Mechanics, vol. 27, no. 9, pp. 1157-1164, 2006.

[11] Y. Yao, Y.-C. Liou, and J.-C. Yao, "Convergence theorem for equilibrium problems and fixed point problems of infinite family of nonexpansive mappings," Fixed Point Theory and Applications, vol. 2007, Article ID 64363, 12 pages, 2007.

[12] S. Plubtieng and R. Punpaeng, "A general iterative method for equilibrium problems and fixed point problems in Hilbert spaces," Journal of Mathematical Analysis and Applications, vol. 336, no. 1, pp. 455469, 2007.

[13] A. Tada and W. Takahashi, "Strong convergence theorem for an equilibrium problem and a nonexpansive mapping," in Nonlinear Analysis and Convex Analysis, W. Takahashi and T. Tanaka, Eds., pp. 609-617, Yokohama, Yokohama, Japan, 2007.

[14] M. A. Noor, "Fundamentals of equilibrium problems," Mathematical Inequalities E Applications, vol. 9, no. 3, pp. 529-566, 2006.

[15] Y. Yao, M.A. Noor, and Y-C. Liou, "On iterative methods for equilibrium problems," Nonlinear Analysis, vol. 70, pp. 497-509, 2009.

[16] L.-C. Zeng and J.-C. Yao, "A hybrid iterative scheme for mixed equilibrium problems and fixed point problems," Journal of Computational and Applied Mathematics, vol. 214, no. 1, pp. 186-201, 2008.

[17] Y. Yao, M. A. Noor, S. Zainab, and Y.-C. Liou, "Mixed equilibrium problems and optimization problems," Journal of Mathematical Analysis and Applications, vol. 354, no. 1, pp. 319-329, 2009.

[18] Y. Yao, H. Zhou, and Y.-C. Liou, "Weak and strong convergence theorems for an asymptotically $k$ strict pseudocontraction and a mixed equilibrium problem," Journal of the Korean Mathematical Society, vol. 46, pp. 561-576, 2009.

[19] P.-E. Mainge, "Regularized and inertial algorithms for common fixed points of nonlinear operators," Journal of Mathematical Analysis and Applications, vol. 344, no. 2, pp. 876-887, 2008.

[20] Y. Yao, Y.-C. Liou, and J.-C. Yao, "An iterative algorithm for approximating convex minimization problem," Applied Mathematics and Computation, vol. 188, no. 1, pp. 648-656, 2007.

[21] G. Marino, V. Colao, L. Muglia, and Y. Yao, "Krasnoselski-Mann iteration for hierarchical fixed points and equilibrium problem," Bulletin of the Australian Mathematical Society, vol. 79, pp. 187-200, 2009.

[22] P.-E. Mainge and A. Moudafi, "Coupling viscosity methods with the extragradient algorithm for solving equilibrium problems," Journal of Nonlinear and Convex Analysis, vol. 9, no. 2, pp. 283-294, 2008.

[23] A. Moudafi, "Weak convergence theorems for nonexpansive mappings and equilibrium problems," Journal of Nonlinear and Convex Analysis, vol. 9, no. 1, pp. 37-43, 2008.

[24] L.-C. Ceng, S. Al-Homidan, Q. H. Ansari, and J.-C. Yao, "An iterative scheme for equilibrium problems and fixed point problems of strict pseudo-contraction mappings," Journal of Computational and Applied Mathematics, vol. 223, no. 2, pp. 967-974, 2009.

[25] G. Marino and H.-K. Xu, "Weak and strong convergence theorems for strict pseudo-contractions in Hilbert spaces," Journal of Mathematical Analysis and Applications, vol. 329, no. 1, pp. 336-346, 2007.

[26] L.-C. Zeng, N.-C. Wong, and J.-C. Yao, "Strong convergence theorems for strictly pseudocontractive mappings of Browder-Petryshyn type," Taiwanese Journal of Mathematics, vol. 10, no. 4, pp. 837-849, 2006.

[27] H. Zhou, "Convergence theorems of common fixed points for a finite family of Lipschitz pseudocontractions in Banach spaces," Nonlinear Analysis: Theory, Methods E Applications, vol. 68, no. 10, pp. 2977-2983, 2008.

[28] I. V. Konnov, "Generalized monotone equilibrium problems and variational inequalities," in Handbook of Generalized Convexity and Generalized Monotonicity, N. Hadjisavvas, S. Komlosi, and S. Schaible, Eds., Springer, New York, NY, USA, 2005. 